\title{
Electromagnetic Scattering from Vibrating Penetrable Objects Using a General Class of Time-Varying Sheet Boundary Conditions
}

\author{
Daniel E. Lawrence, Member, IEEE, and Kamal Sarabandi, Fellow, IEEE
}

\begin{abstract}
Calculation of electromagnetic (EM) scattering from vibrating penetrable cylinders of arbitrary cross-section is presented using a general class of time-varying sheet boundary conditions (SBCs) in conjunction with the method of moments (MoM). Sheet impedance and admittance expressions are first derived from the exact scattering solution for a penetrable circular cylinder with perturbed radius. Then, using the SBCs, integral equations are derived and solved numerically so that vibrating cylinders with arbitrary cross-section can be treated. Cylinder vibrations are assumed to be non-relativistic, allowing a simplified calculation of the scattered Doppler spectrum. A critical factor in the calculation of the potentially small Doppler components is that the time-varying nature of the cylinder boundary, contained within the sheet impedance and admittance expressions, can be isolated from the unperturbed terms in the scattered field. Comparison with exact and analytical perturbation solutions are presented to demonstrate the accuracy of the numerical solution.
\end{abstract}

Index Terms-Doppler spectrum, electromagnetic scattering, impedance boundary conditions, method of moments (MoM), RCS, sheet boundary conditions.

\section{INTRODUCTION}

$\mathbf{I}$ $\mathrm{N}$ a previous paper by the authors [1], a numerical approach is introduced to calculate the electromagnetic (EM) scattering from vibrating perfect electric conducting (PEC) objects using time-varying impedance boundary conditions (IBCs). Movement of the PEC boundary is accounted for by applying a time-varying surface impedance at the unperturbed (stationary) boundary. Unfortunately, the numerical approach in [1] is limited to vibrating PEC bodies. In this paper, we generalize this technique so that vibrating, penetrable objects can be treated.

An electromagnetic (EM) wave scattered from a vibrating target will contain a spectrum of frequencies, referred to as the Doppler spectrum, due to the time-varying nature of the object's boundary. The Doppler spectrum is sometimes called the micro-Doppler in order to differentiate it from the bulk Doppler shift associated with a target moving at constant velocity. There are a number of applications in which the scattered EM Doppler spectrum is of interest. Several recent studies have found that the Doppler spectrum is useful for target detection and recognition [2]-[5]. It has also been applied to modal analysis of vibrating structures [6]. Some early studies involving the calculation of

Manuscript received May 20, 2002; revised March 8, 2005.

D. E. Lawrence is with EMAG Technologies, Inc., Ann Arbor, MI 48108 USA (e-mail: dlawrence@phaseiv.com).

K. Sarabandi is with the Radiation Laboratory, Department of Electrical Engineering and Computer Science, The University of Michigan, Ann Arbor, MI 48109-2122 USA.

Digital Object Identifier 10.1109/TAP.2006.877155
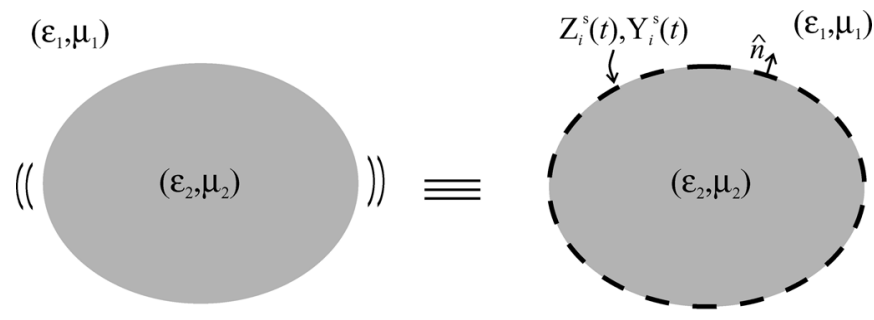

Vibrating Penetrable Object
Stationary Object w/ Time-varying SBCs

Fig. 1. Modeling of vibrating penetrable object with a stationary object having time-varying sheet impedance, $Z_{i}^{s}(t)$, and admittance, $Y_{i}^{s}(t)$. $(i=1,2)$.

EM scattering from oscillating and rotating objects are found in [7]-[11]. None of these studies, however, consider arbitrary vibrations of the surface. More recently, an analytical study of EM scattering from metallic and dielectric circular cylinders with arbitrary vibration has been presented using a perturbation technique [12], [13], but the technique cannot be applied to objects of arbitrary cross-section. What is needed now is an efficient numerical method to calculate the EM scattering from a vibrating, penetrable object having arbitrary cross-section and arbitrary vibration.

As mentioned in [1], a rigorous calculation of the scattering from vibrating objects must include all of the relativistic effects generated by the moving surfaces. For many practical applications, relativistic effects can be ignored and a simplified method for calculating the scattered Doppler spectrum is possible. In the simplified approach, commonly referred to as the quasi-stationary (QS) approximation [14], the scattered field is calculated as if the object were stationary at any time $t$. The scattered field then varies as $t$ is changed. The QS approximation is valid provided that the object is not moving at relativistic speeds and the vibration frequency is much smaller than the EM frequency. A "brute force" application of the QS approximation requires re-discretization of the object and computation of the scattered field for each time instant. In this paper, however, we present a more efficient application of the QS approximation where the object is only discretized once, and a general class of time-varying boundary conditions is derived and applied at the unperturbed boundary to account for the object vibration, see Fig. 1. It will be shown that the time-varying nature of the boundary is contained within the sheet impedance/admittance expressions and can be isolated from the unperturbed component of the scattered field, resulting in an accurate calculation of the Doppler spectrum no matter how small the vibration. 
The literature associated with traditional IBCs is quite large, and a good overview of the subject can be found in [15], [16]. The simplest form is the first-order (standard) impedance boundary condition (SIBC) usually attributed to Leontovich [17]. Generalized IBCs (GIBCs) which incorporate higher derivatives of the surface fields are also possible and usually provide greater accuracy [18]. In this paper, we generalize the IBC expressions to include fields both interior and exterior to a penetrable scatterer. The new boundary conditions are shown to be closely related to those defining a resistive or conducting sheet [15], [19]. Our aim here is not to completely replace the scatterer with an IBC or sheet condition, but instead we retain the original object and require the boundary condition to accurately model the vibration of the object when applied at the object's stationary, unperturbed boundary. The remainder of this paper is organized as follows: A general class of boundary conditions is introduced in Section II, and the relevant sheet impedance/admittance expressions are derived from the scattering solution for a two-dimensional (2-D) dielectric circular cylinder with perturbed radius. Integral equations using the boundary conditions are presented in Section III and solved using the method of moments (MoM) [20]. Finally, results from the numerical solution and comparisons with existing analytical solutions are presented in Section IV.

\section{SHEET BOUNDARY CONDITIONS}

Normally, IBCs are used to simplify a scattering problem by replacing a complex scatterer, such as a penetrable object or coated metallic object, with a surface impedance applied at the exterior boundary. The usual form of the SIBC is given by

$$
\hat{n} \times \mathbf{E}=Z^{s} \hat{n} \times(\hat{n} \times \mathbf{H})
$$

where $Z^{s}$ represents the surface impedance relating the tangential electric and magnetic fields at the boundary. In the present case, however, completely replacing a penetrable object with a surface impedance is not appropriate. Instead we propose to keep the original object, allowing fields internal to the scatterer, and require that the boundary condition applied at the unperturbed boundary account for the perturbation of the object. Consequently, a more general form of the IBC in (1) is needed that includes fields both internal and external to the scatterer. An appropriate set of boundary conditions can be obtained by introducing a general impedance/admittance sheet at the unperturbed boundary. For the scenario shown in Fig. 1, the following set of sheet boundary conditions (SBCs) is postulated:

$$
\begin{aligned}
\hat{n} \times\left(\mathbf{E}_{1}-\mathbf{E}_{2}\right) & =\hat{n} \times \hat{n} \times\left(Z_{1}^{s} \mathbf{H}_{1}+Z_{2}^{s} \mathbf{H}_{2}\right) \\
-\hat{n} \times\left(\mathbf{H}_{1}-\mathbf{H}_{2}\right) & =\hat{n} \times \hat{n} \times\left(Y_{1}^{s} \mathbf{E}_{1}+Y_{2}^{s} \mathbf{E}_{2}\right)
\end{aligned}
$$

where $Z_{i}^{s}$ and $Y_{i}^{s}$ represent the sheet impedance and admittance, respectively, in the $i$ th region $(i=1,2)$. The SBCs of (2) and (3) are closely related to the conditions defining resistive and conducting sheets [19], but they represent a more general form of the sheet conditions. An illustration of the relationship between the SBCs and the traditional sheet conditions is given in Appendix A. It is interesting to note that (2) and (3) reduce to the standard IBC of (1) if the fields are set to zero in either region. Furthermore, if $Z_{i}^{s}=0$ and $Y_{i}^{s}=0$ in both regions, (2) and (3) reduce to the usual boundary conditions at a source-free interface. More general expressions can be obtained by using higher derivatives of the tangential fields on the boundary and will be introduced later in this section.

In what follows, the exact solution for a circular cylinder will be used to derive the sheet impedance/admittance values for a perturbed, penetrable cylinder for both transverse magnetic (TM) and transverse electric (TE) polarizations. The goal is to accurately reproduce the fields scattered from a perturbed cylinder using the SBCs of (2) and (3) applied at the unperturbed boundary. Although derived for a circular cylinder, the SBCs can ultimately be applied to cylinders of arbitrary cross-section and perturbation, following the same approach taken by traditional IBCs.

\section{A. TM Case}

Consider a time-harmonic, TM-polarized plane wave incident upon a penetrable circular cylinder with unperturbed radius $a$

$$
E_{z}^{i}=e^{-j k_{1} x}=\sum_{n=-\infty}^{\infty} j^{-n} J_{n}\left(k_{1} \rho\right) e^{j n \phi} .
$$

The eigenfunction solution for the scattered fields in Region 1 takes the form [21]

$$
E_{1 z}^{s}=\sum_{n=-\infty}^{\infty} j^{-n} a_{n} H_{n}^{(2)}\left(k_{1} \rho\right) e^{j n \phi}
$$

where the mode coefficients, $a_{n}$, are given by

$$
a_{n}=\frac{\frac{Z_{1}}{Z_{2}} J_{n}\left(k_{1} a\right) J_{n}^{\prime}\left(k_{2} a\right)-J_{n}^{\prime}\left(k_{1} a\right) J_{n}\left(k_{2} a\right)}{J_{n}\left(k_{2} a\right) H_{n}^{(2) \prime}\left(k_{1} a\right)-\frac{Z_{1}}{Z_{2}} J_{n}^{\prime}\left(k_{2} a\right) H_{n}^{(2)}\left(k_{1} a\right)} .
$$

In these expressions, $k_{1}$ and $k_{2}$ are the propagation constants and $Z_{1}$ and $Z_{2}$ are the intrinsic impedances of the background and cylinder, respectively. If the radius of the cylinder is perturbed by a small number $\delta$, the perturbed mode coefficients can be obtained by replacing $a$ with $a+\delta$ in (6) followed by a first order Taylor series expansion of each term about $\delta=0$. The perturbed mode coefficients then become (7), shown at the bottom of the page. The approximation in (7) is valid provided that $\delta \ll a, \lambda_{1}$.

$$
\tilde{a}_{n} \approx \frac{\frac{Z_{1}}{Z_{2}}\left[J_{n}\left(k_{1} a\right)+k_{1} \delta J_{n}^{\prime}\left(k_{1} a\right)\right]\left[J_{n}^{\prime}\left(k_{2} a\right)+k_{2} \delta J_{n}^{\prime \prime}\left(k_{2} a\right)\right]-\left[J_{n}^{\prime}\left(k_{1} a\right)+k_{1} \delta J_{n}^{\prime \prime}\left(k_{1} a\right)\right]\left[J_{n}\left(k_{2} a\right)+k_{2} \delta J_{n}^{\prime}\left(k_{2} a\right)\right]}{\left[J_{n}\left(k_{2} a\right)+k_{2} \delta J_{n}^{\prime}\left(k_{2} a\right)\right]\left[H_{n}^{(2) \prime}\left(k_{1} a\right)+k_{1} \delta H_{n}^{(2) \prime \prime}\left(k_{1} a\right)\right]-\frac{Z_{1}}{Z_{2}}\left[J_{n}^{\prime}\left(k_{2} a\right)+k_{2} \delta J_{n}^{\prime \prime}\left(k_{2} a\right)\right]\left[H_{n}^{(2)}\left(k_{1} a\right)+k_{1} \delta H_{n}^{(2) \prime}\left(k_{1} a\right)\right]}
$$


Now if we apply the proposed SBCs in (2) and (3) to the eigenfunction scattering solution for a penetrable circular cylinder of radius $a$, the mode coefficients become (8), shown at the bottom of the page. By equating (8) to the mode coefficients for the perturbed cylinder in (7) and keeping only first order terms in $\delta$, we can solve for sheet impedance and admittance values that will yield an equivalent solution. Following some lengthy algebraic manipulation, and using the relationship from Bessel's equation, it can be shown that an equivalent solution (to first order in $\delta$ ) is obtained when

$$
\begin{aligned}
Z_{1}^{s} & =-j k_{1} \delta Z_{1} \\
Z_{2}^{s} & =+j k_{2} \delta Z_{2} \\
Y_{1}^{s} & =\frac{-j k_{1} \delta}{Z_{1}}\left[1-\left(\frac{n}{k_{1} a}\right)^{2}\right] \\
Y_{2}^{s} & =\frac{+j k_{2} \delta}{Z_{2}}\left[1-\left(\frac{n}{k_{2} a}\right)^{2}\right] .
\end{aligned}
$$

Note that the sheet admittance expressions in (11) and (12) depend on the mode number $n$ and, as such, cannot be applied to a cylinder with non-circular cross-section. This mode dependence suggests that a higher order boundary condition should be used in place of (3). If we revise the general, second order GIBC found in [1], [15] to account for fields interior to the scatterer, we can replace the SBC of (3) with

$$
\begin{aligned}
-\hat{n} \times\left(\mathbf{H}_{1}-\mathbf{H}_{2}\right)=\hat{n} \times \hat{n} \times\left[\gamma_{1} \mathbf{E}_{1}\right. & +\frac{\partial}{\partial s}\left(A_{1} \frac{\partial}{\partial s} \mathbf{E}_{1}\right)+\gamma_{2} \mathbf{E}_{2} \\
& \left.+\frac{\partial}{\partial s}\left(A_{2} \frac{\partial}{\partial s} \mathbf{E}_{2}\right)\right]
\end{aligned}
$$

where $\partial / \partial s$ denotes differentiation tangent to the surface. The increased accuracy of this boundary condition comes from the inclusion of higher order derivatives of the fields. When applied to a circular cylinder with sheet admittance independent of $\phi$, (13) becomes

$H_{1 \phi}-H_{2 \phi}=\left[\gamma_{1}+A_{1} \frac{1}{a^{2}} \frac{\partial^{2}}{\partial \phi^{2}}\right] E_{1 z}+\left[\gamma_{2}+A_{2} \frac{1}{a^{2}} \frac{\partial^{2}}{\partial \phi^{2}}\right] E_{2 z}$

When the boundary condition in (14) is compared with (3) together with (11) and (12), and recalling that $E_{z}$ has a $\phi$-dependence of $e^{j n \phi}$, it becomes evident that an equivalent solution (to first order in $\delta$ ) for the perturbed mode coefficients is achieved if

$$
\begin{array}{ll}
\gamma_{1}=\frac{-j k_{1} \delta}{Z_{1}}, & \gamma_{2}=\frac{+j k_{2} \delta}{Z_{2}} \\
A_{1}=\frac{-j \delta}{k_{1} Z_{1}}, & A_{2}=\frac{+j \delta}{k_{2} Z_{2}} .
\end{array}
$$

This completes the derivation of the sheet impedance/admittance values for TM polarization. Although derived for a circular cylinder with perturbed radius, the boundary conditions can be extended for arbitrary perturbation of a cylinder by allowing $\delta$ to be a function of position on the boundary. Furthermore, a cylinder of arbitrary cross-section can be treated by using the boundary conditions with a numerical technique such as MoM. Details of the numerical approach are presented in Section III.

\section{B. TE Case}

Before applying the numerical technique, it should be noted that the sheet impedance/admittance values for TE incidence can be easily derived using the duality of the field equations. Note that the boundary conditions in (2) and (3) are duals of each other with an interchange of $Z_{i}^{s}$ and $Y_{i}^{s}$ and applied to the surface of a perturbed boundary to yield the dual boundary conditions.

\section{INTEGRAL EQUATION FORMULATION}

In this section, we develop integral equations incorporating the SBCs of the previous section that allows us to solve for the scattering from a perturbed, penetrable cylinder of arbitrary cross-section for the TM and TE cases.

\section{A. TM Case}

Consider a TM-polarized plane wave incident at $\phi^{i}$

$$
E_{z}^{i}=e^{-j k_{1}\left(x \cos \phi^{i}+y \sin \phi^{i}\right)} .
$$

In order to derive the relevant integral equations, the perturbed cylinder is replaced with a closed surface coincident with the unperturbed cylinder boundary. Invoking the equivalence principle, two sets of electric $\left(\mathbf{J}_{1}\right.$ and $\left.\mathbf{J}_{2}\right)$ and magnetic ( $\mathbf{M}_{1}$ and $\mathbf{M}_{2}$ ) equivalent currents are introduced on the unperturbed boundary, see Fig. 2. Each set radiates in an infinite homogenous medium with constitutive parameters corresponding to the associated region. The equivalent currents are related to the tangential fields on the boundary by

$$
\begin{aligned}
\hat{n} \times \mathbf{H}_{1} & =\mathbf{J}_{1}, \quad-\hat{n} \times \mathbf{H}_{2}=\mathbf{J}_{2} \\
\mathbf{E}_{1} \times \hat{n} & =\mathbf{M}_{1}, \quad-\mathbf{E}_{2} \times \hat{n}=\mathbf{M}_{2} .
\end{aligned}
$$

Two integral equations are obtained by enforcing the $E$-field conditions in (19)

$$
\begin{aligned}
E_{1 z} & =E_{z}^{i}+E_{1 z}^{s}=M_{1 s} \\
-E_{2 z} & =-E_{2 z}^{s}=M_{2 s}
\end{aligned}
$$

$$
=\frac{\frac{Z_{1}}{Z_{2}}\left[J_{n}\left(k_{1} a\right)+\frac{j Z_{1}^{s}}{Z_{1}} J_{n}^{\prime}\left(k_{1} a\right)\right]\left[J_{n}^{\prime}\left(k_{2} a\right)+j Y_{2}^{s} Z_{2} J_{n}\left(k_{2} a\right)\right]-\left[J_{n}^{\prime}\left(k_{1} a\right)-j Y_{1}^{s} Z_{1} J_{n}\left(k_{1} a\right)\right]\left[J_{n}\left(k_{2} a\right)-\frac{j Z_{2}^{s}}{Z_{2}} J_{n}^{\prime}\left(k_{2} a\right)\right]}{\left[J_{n}\left(k_{2} a\right)-\frac{j Z_{2}^{s}}{Z_{2}} J_{n}^{\prime}\left(k_{2} a\right)\right]\left[H_{n}^{(2) \prime}\left(k_{1} a\right)-j Y_{1}^{s} Z_{1} H_{n}^{(2)}\left(k_{1} a\right)\right]-\frac{Z_{1}}{Z_{2}}\left[J_{n}^{\prime}\left(k_{2} a\right)+j Y_{2}^{s} Z_{2} J_{n}\left(k_{2} a\right)\right]\left[H_{n}^{(2)}\left(k_{1} a\right)+\frac{j Z_{1}^{s}}{Z_{1}} H_{n}^{(2) \prime}\left(k_{1} a\right)\right]}
$$




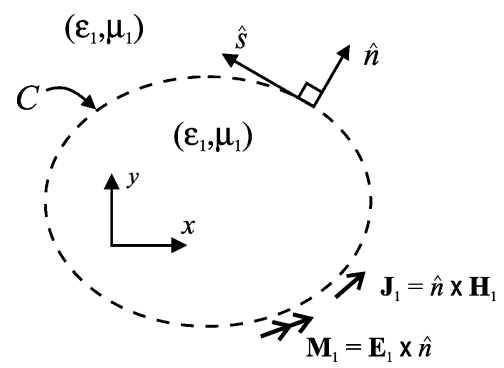

Equivalence for Region 1

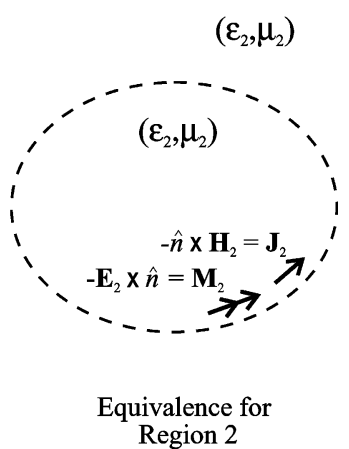

Region 2
Fig. 2. Geometry of scattering problem for cylinder of arbitrary cross-section.

where the scattered fields are given by

$$
\begin{aligned}
E_{i z}^{s}(\bar{\rho})=-\frac{Z_{i} k_{i}}{4} \int_{C} J_{i z}\left(\bar{\rho}^{\prime}\right) H_{0}^{(2)}\left(k_{i}\left|\bar{\rho}-\bar{\rho}^{\prime}\right|\right) d s^{\prime} \\
-\frac{j}{4} \int_{C} M_{i s}\left(\bar{\rho}^{\prime}\right) \frac{d}{d n^{\prime}} H_{0}^{(2)}\left(k_{i}\left|\bar{\rho}-\bar{\rho}^{\prime}\right|\right) d s^{\prime}
\end{aligned}
$$

with $i=1,2$. The operator $d / d n^{\prime}$ denotes differentiation along the direction normal to $C$, and the differential element $d s^{\prime}$ represents arc length along the integration contour. Coupling of the two integral equations is achieved through the SBCs of Section II. For the TM case, we can rewrite the boundary conditions of (2) and (13) using equivalent currents

$$
\begin{aligned}
M_{1 s}+M_{2 s}= & Z_{1}^{s} J_{1 z}-Z_{2}^{s} J_{2 z} \\
J_{1 z}+J_{2 z}= & \gamma_{1} M_{1 s}+\frac{\partial}{\partial s}\left(A_{1} \frac{\partial}{\partial s} M_{1 s}\right) \\
& -\gamma_{2} M_{2 s}-\frac{\partial}{\partial s}\left(A_{2} \frac{\partial}{\partial s} M_{2 s}\right) .
\end{aligned}
$$

By solving the system of equations, the equivalent currents in Region 2 can be written in terms of the currents in Region 1. Keeping only first order terms in $\delta$

$$
\begin{aligned}
M_{2 s}= & -M_{1 s}+\left(Z_{1}^{s}+Z_{2}^{s}\right) J_{1 z} \\
J_{2 z}= & -J_{1 z}+\left(\gamma_{1}+\gamma_{2}\right) M_{1 s} \\
& +\frac{\partial}{\partial s}\left(\left(A_{1}+A_{2}\right) \frac{\partial}{\partial s} M_{1 s}\right) .
\end{aligned}
$$

Now substituting (25) and (26) into (21) yields two integral equations, (20) and (21), for the two unknown current components $J_{1 z}$ and $M_{1 s}$.

The coupled integral equations are solved using the traditional pulse-basis and point-matching MoM technique. The contour $C$ is discretized into $N$ linear segments, and on each segment the current distributions of $J_{1 z}$ and $M_{1 s}$ are assumed constant. Enforcing the integral equations (20) and (21) at the midpoint of each segment results in the matrix equation

$$
\left[\begin{array}{cc}
Z_{m n}^{11} & Z_{m n}^{12} \\
Z_{m n}^{21}+\delta Z_{m n}^{21} & Z_{m n}^{22}+\delta Z_{m n}^{22}
\end{array}\right]\left[\begin{array}{c}
J_{n} \\
M_{n}
\end{array}\right]=\left[\begin{array}{c}
V_{m} \\
0
\end{array}\right]
$$

where $J_{n}$ and $M_{n}$ are $N \times 1$ vectors denoting the electric and magnetic currents, respectively, on the $n$th segment. $V_{m}$ is the $N \times 1$ excitation vector whose elements are

$$
V_{m}=E_{z}^{i}\left(\bar{\rho}_{m}\right)
$$

where $\bar{\rho}_{m}$ denotes the midpoint of the $m$ th segment. Finally, each component of the impedance matrix is an $N \times N$ matrix whose elements are given by

$$
\begin{aligned}
& Z_{m n}^{11}= \frac{Z_{1} k_{1}}{4} \int_{C_{n}} H_{0}^{(2)}\left(k_{1}\left|\bar{\rho}_{m}-\bar{\rho}^{\prime}\right|\right) d s^{\prime} \\
& Z_{m n}^{21}= \frac{Z_{2} k_{2}}{4} \int_{C_{n}} H_{0}^{(2)}\left(k_{2}\left|\bar{\rho}_{m}-\bar{\rho}^{\prime}\right|\right) d s^{\prime} \\
& Z_{m n}^{12}= \mathrm{I}_{m n}+\frac{j}{4} \int_{C_{n}} \frac{d}{d n^{\prime}} H_{0}^{(2)}\left(k_{1}\left|\bar{\rho}_{m}-\bar{\rho}^{\prime}\right|\right) d s^{\prime} \\
& Z_{m n}^{22}=-\mathrm{I}_{m n}+\frac{j}{4} \int_{C_{n}} \frac{d}{d n^{\prime}} H_{0}^{(2)}\left(k_{2}\left|\bar{\rho}_{m}-\bar{\rho}^{\prime}\right|\right) d s^{\prime} \\
& \delta Z_{m n}^{21}= {\left[Z_{1}^{s}\left(\bar{\rho}_{m}\right)+Z_{2}^{s}\left(\bar{\rho}_{m}\right)\right] \mathrm{I}_{m n} } \\
&-\frac{j}{4} \int_{C_{n}} \frac{d}{d n^{\prime}}\left[Z_{1}^{s}\left(\bar{\rho}_{m}\right)+Z_{2}^{s}\left(\bar{\rho}_{m}\right)\right] \\
& \delta Z_{m n}^{22}=-\frac{Z_{2} k_{2}}{4} \int_{C_{n}}\left[\gamma_{1}\left(\bar{\rho}_{m}\right)+\gamma_{2}\left(\bar{\rho}_{m}\right)\right] H_{0}^{(2)}\left(k_{2}\left|\bar{\rho}_{m}-\bar{\rho}^{\prime}\right|\right) d s^{\prime} \\
&+\frac{Z_{2} k_{2}}{4} \int_{C_{n}} \frac{d}{d s^{\prime}}\left(\left[A_{1}\left(\bar{\rho}_{m}\right)+A_{2}\left(\bar{\rho}_{m}\right)\right] d s^{\prime}\right. \\
&\left.\times \frac{d}{d s^{\prime}} H_{0}^{(2)}\left(k_{2}\left|\bar{\rho}_{m}-\bar{\rho}^{\prime}\right|\right)\right) d s^{\prime}
\end{aligned}
$$

where $\mathrm{I}_{m n}$ are elements of the identity matrix. Note that components of the impedance matrix containing the sheet impedance/ admittance expressions are preceded by $\delta$ to denote that they are small quantities. Once the currents are found by solving (27), the scattered field $E_{1 z}^{s}$ can easily be found by evaluating (22).

\section{B. TE Case}

The numerical formulation for the TE case can be easily found by utilizing the duality relationship of the field equations.

\section{NUMERICAL RESUltS}

In order to illustrate the validity of the numerical solution presented here, several canonical cases are considered. The exact solution for a circular cylinder with a perturbed radius provides a benchmark for comparison. Note, however, that the numerical solution allows the application to other, non-canonical cases with arbitrary perturbation. The time-varying boundary conditions for a vibrating cylinder are also introduced in this section, and results are compared with known analytical solutions published in [13]. 


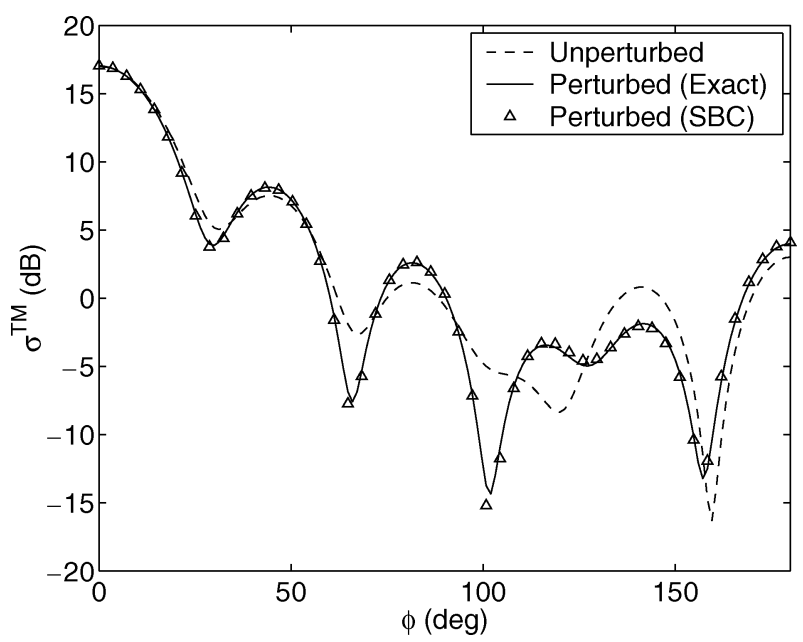

Fig. 3. SBC approximation of TM scattering from circular dielectric cylinder with a perturbed radius. $\left(\epsilon_{2}=2.2 \epsilon_{1}, a=0.75 \lambda_{1}, \delta=0.02 \lambda_{1}\right)$.

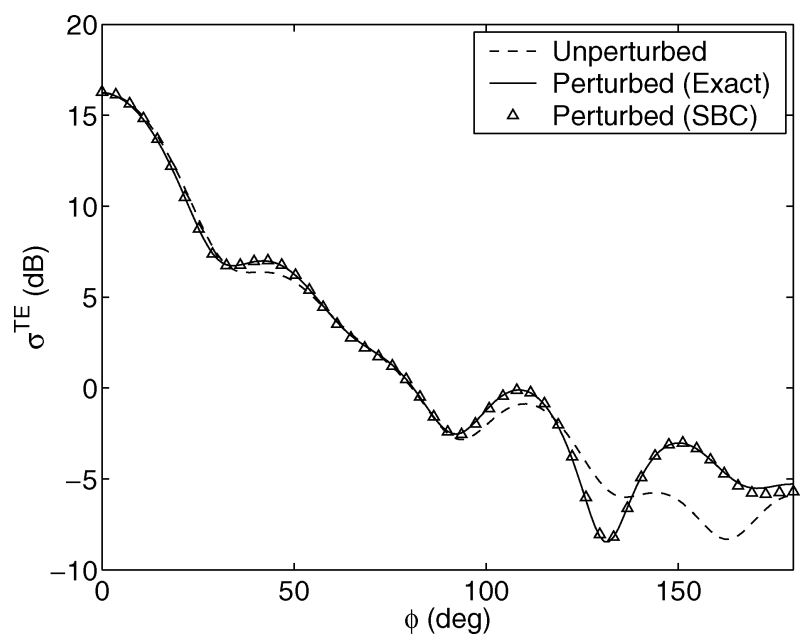

Fig. 4. SBC approximation of TE scattering from circular dielectric cylinder with a perturbed radius. $\left(\epsilon_{2}=2.2 \epsilon_{1}, a=0.75 \lambda_{1}, \delta=0.02 \lambda_{1}\right)$.

\section{A. Perturbed Cylinder}

For a dielectric circular cylinder, an exact scattering solution is known for both an unperturbed cylinder with radius $a$ and a perturbed cylinder with radius $a+\delta$. The sheet boundary conditions are applied at the unperturbed boundary but should accurately predict the scattering from the perturbed cylinder. As an example, consider a plane wave incident at $\phi^{i}=0^{\circ}$ upon a dielectric cylinder with dielectric constant $\epsilon_{2}=2.2 \epsilon_{1}$ and radius $a=0.75 \lambda_{1}$ perturbed by $\delta=0.02 \lambda_{1}$. The background medium (Region 1) is assumed to be free space. Figs. 3 and 4 show the bistatic RCS for the TM and TE cases, respectively. The 2-D RCS is calculated

$$
\sigma^{T M}=\lim _{\rho \rightarrow \infty}\left[2 \pi \rho \frac{\left|E_{1 z}^{s}\right|^{2}}{\left|E_{z}^{i}\right|^{2}}\right]
$$

for the TM case, and $\sigma^{T E}$ is calculated in similar fashion using $H_{z}$. In Figs. 3 and 4, the SBC approximation is in excellent agreement with the exact solution for a dielectric cylinder with

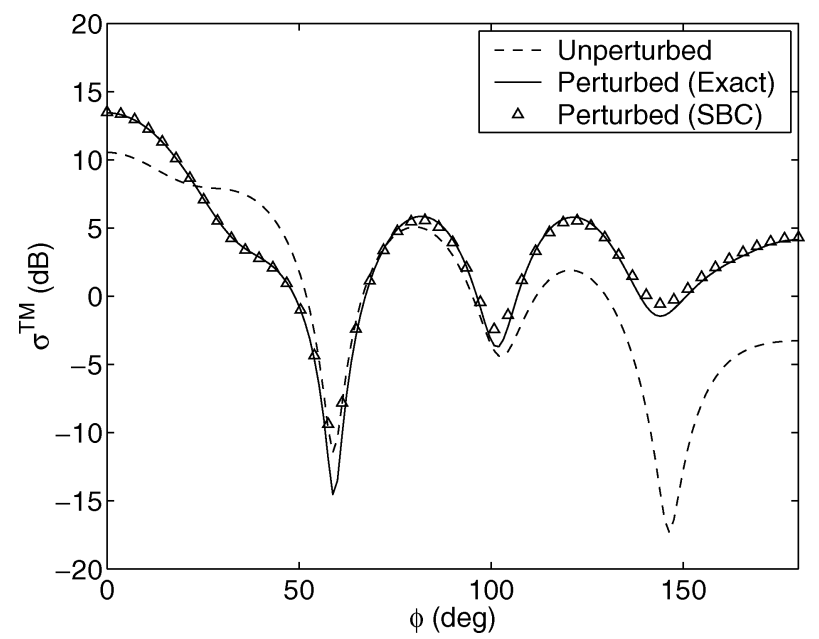

Fig. 5. SBC approximation of TM scattering from circular dielectric cylinder with a perturbed radius. $\left(\epsilon_{2}=10.0 \epsilon_{1}, a=0.75 \lambda_{1}, \delta=0.02 \lambda_{1}\right)$.

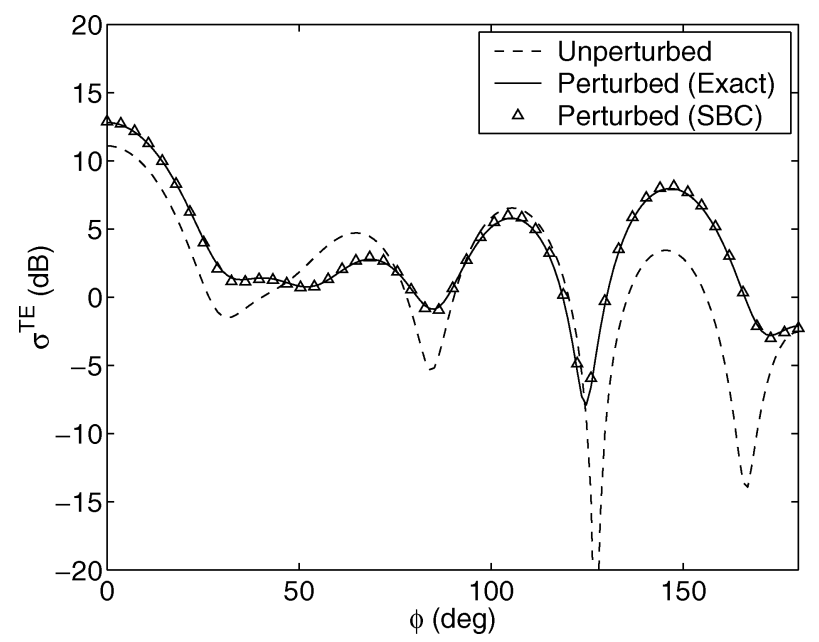

Fig. 6. SBC approximation of TE scattering from circular dielectric cylinder with a perturbed radius. $\left(\epsilon_{2}=10.0 \epsilon_{1}, a=0.75 \lambda_{1}, \delta=0.02 \lambda_{1}\right)$.

perturbed radius. If the dielectric constant of the cylinder is increased, the SBC approximation remains accurate. Consider a cylinder with dielectric constant $\epsilon_{2}=10.0 \epsilon_{1}$ with the same dimension and perturbation as before. Figs. 5 and 6, illustrate the ability of the SBCs to accurately predict the change in RCS caused by the perturbation. Notice that at some scattering angles the change is greater than $10 \mathrm{~dB}$. Similar results are obtained for other dielectric cylinders having different dielectric constant values and different radii.

\section{B. Doppler Spectrum of Vibrating Cylinder}

To model the scattering from a vibrating cylinder, the SBCs considered here must become time-varying. Furthermore, to accurately calculate the Doppler spectrum from the vibrating cylinder it is necessary for the time-varying components of the scattered field to be separated from the constant terms. With this in mind, we revisit the matrix equation of (27) used to solve for the electric and magnetic surface currents. The inverse of the impedance matrix is used to solve the system and needs to be written in such a way that the perturbed and unperturbed terms are isolated from each other. This is accomplished by 
first separating the terms containing the perturbation parameter $\delta$ and then applying the well known matrix identity given by (42) in Appendix B. Keeping only first order terms in $\delta$, we can write

$$
\begin{aligned}
{\left[\begin{array}{cc}
Z_{m n}^{11} & Z_{m n}^{12} \\
Z_{m n}^{21}+\delta Z_{m n}^{21} & Z_{m n}^{22}+\delta Z_{m n}^{22}
\end{array}\right]^{-1} } & =\left[Z_{u}+\delta Z\right]^{-1} \\
& \approx Z_{u}^{-1}+Z_{u}^{-1} \delta Z Z_{u}^{-1}
\end{aligned}
$$

where

$$
Z_{u}=\left[\begin{array}{cc}
Z_{m n}^{11} & Z_{m n}^{12} \\
Z_{m n}^{21} & Z_{m n}^{22}
\end{array}\right] \text { and } \delta Z=\left[\begin{array}{cc}
0 & 0 \\
\delta Z_{m n}^{21} & \delta Z_{m n}^{22}
\end{array}\right]
$$

The first term in (36), $Z_{u}^{-1}$, is the impedance matrix inverse for the unperturbed problem, and all of the perturbed components are isolated in the second term on the right. Note that only the unperturbed impedance matrix needs to be inverted and that no further matrix inversion is required. When the cylinder is vibrating, the time-varying nature of the boundary conditions is contained within the expression for the perturbation of the surface, and as a result, the time-dependence is confined to the perturbed terms in (36). Isolating the time-varying terms is significant because the inverse of the unperturbed impedance matrix need only be computed once. It can then be used to calculate the perturbed, time-varying portion of the scattered field.

To illustrate the scattering from a vibrating object, consider a dielectric cylinder excited at a mechanical resonance. The excitation can be achieved through acoustic or mechanical means. Acoustic excitation of solid elastic cylinders has been treated in [22], and the cylinder displacement derived therein will be used here. The radial displacement at the surface of the cylinder takes the form

$$
u_{\rho}(\phi, t)=\sum_{n=0}^{\infty} U_{n} \cos \left(\omega_{a} t+\theta_{n}\right) \cos n \phi
$$

where $U_{n}$ and $\theta_{n}$ are the magnitude and phase, respectively, associated with the $n$th mode of vibration, and $\omega_{a}$ is the vibration frequency. The $n=2$ mode is the fundamental mode of vibration for a solid cylinder occurring at the lowest resonant frequency. Assuming that this mode is excited, the time-varying perturbation of the surface becomes

$$
\delta(\phi, t)=U_{2} \cos \left(\omega_{a} t+\theta_{2}\right) \cos 2 \phi .
$$

Substituting (38) into the sheet impedance/admittance expressions in Section II we can use the numerical procedure of Section III to solve for the scattered fields. Taking the Fourier transform of the time-varying scattered field provides the scattered Doppler spectrum. An example Doppler spectrum for an object vibrating at frequency $f_{a}$ is given in Fig. 7. The unshifted component of the scattered spectrum, corresponding to the scattered field from the target when stationary, can be obtained from the unperturbed terms in the numerical solution. The 1st harmonic of the Doppler spectrum is shifted in frequency by an amount equal to the vibration frequency and can be calculated from the perturbed (time-varying) terms in the numerical solution.

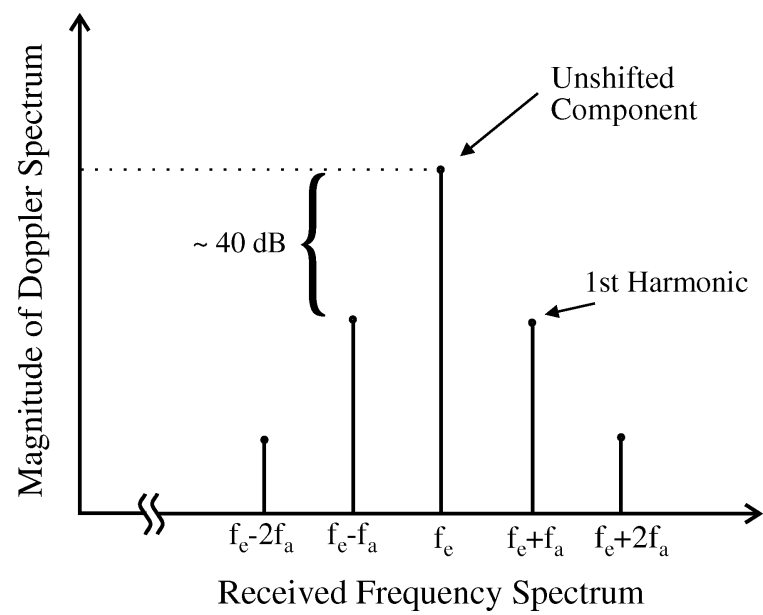

Fig. 7. Example Doppler spectrum scattered from an object vibrating at a frequency $f_{a}$ illuminated by an incident EM frequency $f_{e}$.

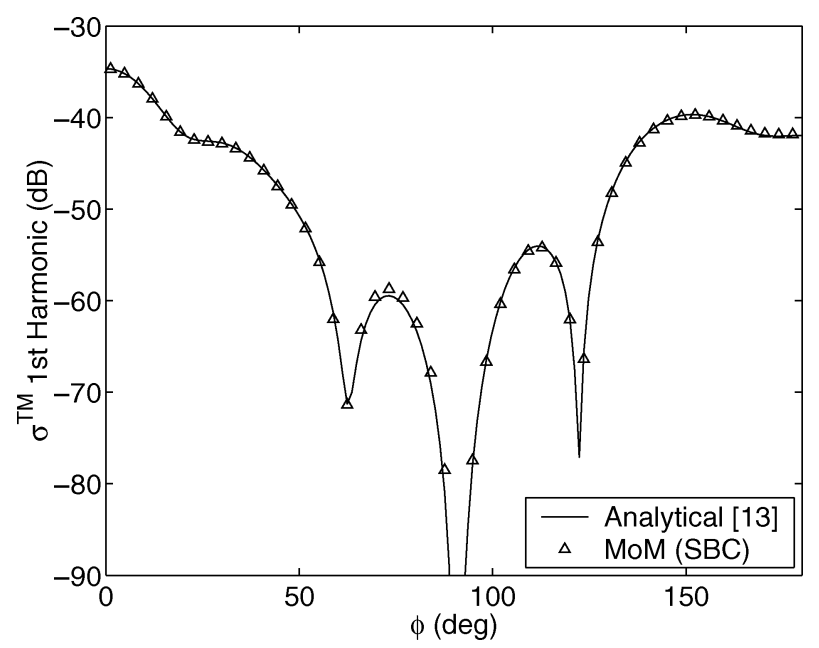

Fig. 8. Comparison of the MoM (SBC) and the analytical perturbation solution for TM Doppler scattering from a vibrating circular dielectric cylinder. The $n=2$ mechanical mode of the cylinder is excited. $\left(a=1.0 \lambda_{1}, \epsilon_{2}=2.0 \epsilon_{1}\right.$, Max displacement $=0.001 \lambda_{1}$ ).

Since the time-varying nature of the object is manifest in the Doppler components of the scattered spectrum, we are interested in the scattering behavior of the 1st harmonic of the Doppler spectrum. For TM incidence upon a dielectric cylinder, $\epsilon_{2}=2.0 \epsilon_{1}$, with radius $a=1.0 \lambda$, the bistatic scattering of the 1st harmonic is calculated using the MoM and shown in Fig. 8. The $n=2$ mechanical mode of the cylinder is excited. The MoM is shown to have excellent agreement with the analytical perturbation solution from [13]. For TE incidence, the bistatic scattering of the 1st harmonic can be seen in Fig. 9 where again the $n=2$ mode is excited. Again, excellent agreement is obtained for all scattering angles.

\section{CONCLUSION}

In this paper, a general class of boundary conditions has been introduced to model EM scattering from vibrating penetrable objects. A MoM implementation of the SBCs is presented and shown to provide an accurate solution for both TM and TE polarizations. Separation of the perturbed, time-varying terms 


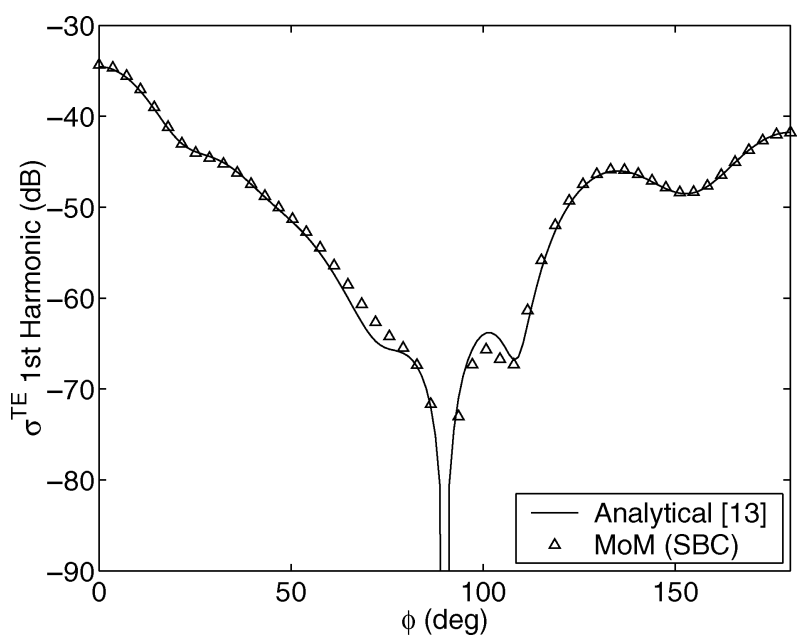

Fig. 9. Comparison of the MoM (SBC) and the analytical perturbation solution for TE Doppler scattering from a vibrating circular cylinder. The $n=$ 2 mechanical mode of the cylinder is excited. $\left(a=1.0 \lambda_{1}, \epsilon_{2}=2.0 \epsilon_{1}\right.$, Max displacement $\left.=0.001 \lambda_{1}\right)$.

in the numerical solution is critical in calculating the small Doppler components of the scattered spectrum. Although a circular cylinder is used for comparison with known analytical results, the MoM with the SBCs derived in this paper can be applied to cylinders of arbitrary cross-section with arbitrary perturbations. The SBCs presented here can also be extended to model 3-D vibrating objects and is reserved for future work.

\section{APPENDIX A \\ RESISTIVE/CONDUCTING SHEET CONDITIONS}

The purpose of this section is to illustrate the relationship between the SBCs presented in this paper and resistive/conducting sheets found previously in the literature. Thin layers of material are often simulated using resistive and/or conducting sheets [15], [19]. In general, combined resistive and conducting sheets are used when both the permittivity and permeability of the thin layer are different from the surrounding medium. The boundary conditions proposed in [19] for a combined sheet are

$$
\begin{aligned}
\hat{n} \times\left(\mathbf{E}^{+}-\mathbf{E}^{-}\right) & =\frac{Z_{0}}{2 R_{m}} \hat{n} \times \hat{n} \times\left(\mathbf{H}^{+}+\mathbf{H}^{-}\right) \\
-\hat{n} \times\left(\mathbf{H}^{+}-\mathbf{H}^{-}\right) & =\frac{Y_{0}}{2 R_{e}} \hat{n} \times \hat{n} \times\left(\mathbf{E}^{+}+\mathbf{E}^{-}\right)
\end{aligned}
$$

where $Z_{0}$ and $Y_{0}$ are the intrinsic impedance and admittance, respectively, of the background medium, and the superscripts $+/-$ denote the field evaluated just above/below the boundary, respectively. The sheet resistivity and conductivity terms are

$$
R_{e}=\frac{-j}{k_{0} \tau\left(\epsilon_{r}-1\right)} \quad \text { and } \quad R_{m}=\frac{-j}{k_{0} \tau\left(\mu_{r}-1\right)}
$$

where $\tau$ is the sheet thickness, $k_{0}$ is the propagation constant of the background medium, and $\epsilon_{r}$ and $\mu_{r}$ are the relative permittivity and permeability, respectively, of the sheet. Note that the conditions in (39) and (40) can be obtained from the more general conditions of (2) and (3) from Section II if the sheet impedance and admittance expressions are related by $Z_{2}^{s}=Z_{1}^{s}$ and $Y_{2}^{s}=Y_{1}^{s}$. The limitation of (39) and (40), however, is that the medium above and below the sheet must be the same. If the sheet is placed on an interface between two different media, the boundary conditions can be derived from the more general conditions in (2) and (3).

\section{APPENDIX B MATRIX IDENTITY}

For square matrices $\mathbf{A}$ and $\mathbf{B}$ such that $\mathbf{A}, \mathbf{A}+\mathbf{B}$, and $\mathbf{I}+$ $\mathbf{B A}^{-1}$ are nonsingular, the following identity holds [23]:

$$
(\mathbf{A}+\mathbf{B})^{-1}=\mathbf{A}^{-1}-\mathbf{A}^{-1}\left(\mathbf{I}+\mathbf{B} \mathbf{A}^{-1}\right)^{-1} \mathbf{B A}^{-1}
$$

where $\mathbf{I}$ is the identity matrix.

\section{REFERENCES}

[1] D. E. Lawrence and K. Sarabandi, "Electromagnetic scattering from vibrating metallic objects using time-varying impedance boundary conditions," IEEE Trans. Antennas Propag., vol. 2, pp. 782-785, Jan. 2002.

[2] V. C. Chen and R. Lipps, "Time-frequency signatures of microDoppler phenomenon for feature extraction," in Proc. SPIE-Int. Soc. for Optical Engineering, 2000, vol. 4056, pp. 220-226.

[3] V. C. Chen, "Analysis of radar micro-Doppler signature with timefrequency transform," in Proc. IEEE Signal Processing Workshop on Statistical Signal and Array Processing, 2000, pp. 463-466.

[4] G. Greneker, J. Geisheimer, and D. Asbell, "Extraction of micro-Doppler from vehicle targets at X-band frequencies," in Proc. SPIE-Int. Soc. for Optical Engineering, 2001, vol. 4374, pp. $1-9$.

[5] D. C. Braunreiter, H. W. Chen, M. L. Cassabaum, J. G. Riddle, A. A. Samuel, J. F. Scholl, and H. A. Schmitt, "On the use of space-time adaptive processing and time-frequency data representations for detection of near-stationary targets in monostatic clutter," in Proc. 10th IEEE Workshop on Statistical Signal and Array Processing, 2000, pp. $472-475$.

[6] M. Martarelli, G. M. Revel, and C. Santolini, "Automated modal analysis by scanning laser vibrometry: problems and uncertainties associated with the scanning system calibration," Mech. Sys. Signal Processing, vol. 15, no. 3, pp. 581-601, May 2001.

[7] S. R. Borkar and R. F. H. Yang, "Reflection of electromagnetic waves from oscillating surfaces," IEEE Trans. Antennas Propag., vol. AP-23, no. 1, pp. 122-127, Jan. 1975.

[8] K. Tanaka, "Scattering of electromagnetic waves by a rotating perfectly conducting cylinder with arbitrary cross section: Point-matching method," IEEE Trans. Antennas Propag., vol. AP-28, no. 6, pp. 796-803, Nov. 1980.

[9] B. M. Petrov, "Spectral characteristic of the scatter field from a rotating impedance cylinder in uniform motion," Radio Eng. Electron. Phys. (USSR), vol. 17, pp. 1431-1437, 1972.

[10] I. J. Lahaie and D. J. Sengupta, "Scattering of electromagnetic waves by a slowly rotating rectangular metal plate," IEEE Trans. Antennas Propag., vol. AP-27, pp. 40-46, Jan. 1979.

[11] C. W. Chuang, "Backscatter of a large rotating conducting cylinder of arbitrary cross section," IEEE Trans. Antennas Propag., vol. AP-27, pp. 92-95, Jan. 1979.

[12] K. Sarabandi and D. E. Lawrence, "Acoustic and electromagnetic wave interaction: Estimation of Doppler spectrum from an acoustically vibrated metallic circular cylinder," IEEE Trans. Antennas Propag., vol. 51, pp. 1499-1507, Jul. 2003.

[13] D. E. Lawrence and K. Sarabandi, "Acoustic and electromagnetic wave interaction: analytical formulation for acousto-electromagnetic scattering behavior of a dielectric cylinder," IEEE Trans. Antennas Propag., vol. 49, no. 10, pp. 1382-1392, Oct. 2001.

[14] J. Van Bladel, "Electromagnetic fields in the presence of rotating bodies," Proc. IEEE, vol. 64, pp. 301-318, Mar. 1976.

[15] T. B. A. Senior and J. L. Volakis, Approximate Boundary Conditions in Electromagnetics. London, U.K.: IEE Press, 1995.

[16] D. J. Hoppe and Y. Rahmat-Samii, Impedance Boundary Conditions in Electromagnetics. Washington, D.C.: Taylor \& Francis, 1995.

[17] M. A. Leontovich, Investigations on Radiowave Propagation-Part II. Moscow, USSR: Academy of Sciences, 1948. 
[18] S. N. Karp and F. C. Karal, Jr., "Generalized impedance boundary conditions with applications to surface wave structures," in Electromagnetic Wave Theory, J. Brown, Ed. New York: Pergamon Press, 1965, pt. 1 , pp. $479-483$.

[19] T. B. A. Senior, "Combined resistive and conductive sheets," IEEE Trans. Antennas Propag., vol. AP-33, no. 5, pp. 577-579, May 1985.

[20] R. F. Harrington, Field Computation by Moment Methods. New York: Macmillan, 1968.

[21] R. F. Harrington, Time-Harmonic Electromagnetic Fields. New York: McGraw-Hill, 1961.

[22] J. J. Faran, "Sound scattering by solid cylinders and spheres," J. Acous. Soc. Amer., vol. 23, no. 4, pp. 405-418, Jul. 1951.

[23] M. D. Srinath, P. K. Rajasekaran, and R. Viswanathan, Introduction to Statistical Signal Processing With Applications. Englewood Cliffs, NJ: Prentice Hall, 1996, pp. 439-440.

Daniel E. Lawrence (S'98-M'02) received the B.E.E. and M.S. degrees in electrical engineering from Auburn University, Auburn, AL, in 1996 and 1998, respectively, and the $\mathrm{Ph} . \mathrm{D}$. degree in electrical engineering from the University of Michigan, Ann Arbor, in 2002.

From 1997 to 2000, he was a DoD Graduate Research Fellow investigating the use of low-frequency magnetic fields for buried landmine discrimination. With the Radiation Lab at the University of Michigan, he developed novel analytical solutions for the interaction of acoustics and electromagnetics applied to buried object detection. Currently, he is with Phase IV Systems, Inc., Huntsville, AL, developing advanced microwave radar for Army vehicular active protection systems (APS).

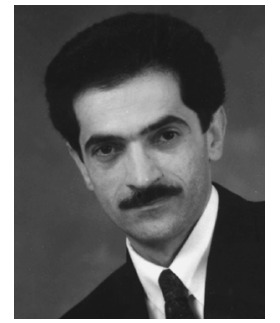

Kamal Sarabandi (S'87-M'90-SM'92-F'00) received the B.S. degree in electrical engineering from Sharif University of Technology, Tehran, Iran, in 1980, the M.S. degree in electrical engineering and mathematics and the Ph.D. degree in electrical engineering both from The University of Michigan-Ann Arbor, in 1986 and 1989, respectively.

$\mathrm{He}$ is Director of the Radiation Laboratory and a Professor with the Department of Electrical Engineering and Computer Science, The University of Michigan. His research areas of interest include microwave and millimeter-wave radar remote sensing, metamaterials, electromagnetic wave propagation, and antenna miniaturization. He has 20 years of experience with wave propagation in random media, communication channel modeling, microwave sensors, and radar systems, and is leading a large research group including two research scientists, ten Ph.D. and two M.S. degree students. Over the past 10 years, he has graduated $22 \mathrm{Ph} . \mathrm{D}$. degree students. He has served as the Principal Investigator on many projects sponsored by NASA, JPL, ARO, ONR, ARL, NSF, DARPA and numerous industries. He has published many book chapters and more than 130 papers in refereed journals on electromagnetic scattering, random media modeling, wave propagation, antennas, metamaterials, microwave measurement techniques, radar calibration, inverse scattering problems, and microwave sensors. He has also had more than 320 papers and invited presentations in many national and international conferences and symposia on similar subjects.

Dr. Sarabandi is a Member of the International Scientific Radio Union (URSI) Commission F and of The Electromagnetic Academy. He received the Henry Russel Award from the Regent of The University of Michigan-Ann Arbor (the highest honor the University of Michigan bestows on a faculty member at the assistant or associate level). He received a 1996 Teaching Excellence Award from the Department of Electrical Engineering and Computer Science and a 1999 GAAC Distinguished Lecturer Award from the German Federal Ministry for Education, Science, and Technology, given to about ten individuals worldwide in all areas of engineering, science, medicine, and law. In 2005, he received the IEEE GRS Distinguished Achievement Award. He also received the 2003/ 2004 College of Engineering Research Excellence Award and the 2005 Faculty Recognition Award, both from The University of Michigan-Ann Arbor. In the past several years, joint papers presented by his students at a number of symposia (IEEE APS'95,'97,'00,'01,'03,'05 IEEE IGARSS'99,'02, IEEE MTTS'01) have received student prize paper awards. He is a Vice President of the IEEE Geoscience and Remote Sensing Society (GRSS), a past Chairman of the Awards Committee of the IEEE GRSS from 1998 to 2002, and a Member of the IEEE Technical Activities Board Awards Committee from 2000 to 2002. He was an Associate Editor of the IEEE TRANSACTIONS ON ANTENNAS AND PROPAGATION and the the IEEE SENSORS JOURNAL. He is listed in American Men \& Women of Science, Who's Who in America, and Who's Who in Electromagnetics. 